\title{
Ebbinghaus Illusion
}

\section{Relative Size as a Possible Invariant Under Technically Varied Conditions?}

\author{
John H. Krantz \\ Department of Psychology, Hanover College, Hanover, IN, USA
}

\begin{abstract}
For 25 years, the web has been used for psychological research (Krantz et al., 1997; Reips, 1997). While many areas of psychology have benefitted from the increased access to participants and other benefits of web-based research, one area of psychology has rarely taken advantage of the online format, that is, sensation and perception. Largely, sensation and perception research has not used the web because of the need for carefully calibrated equipment to successfully run their experiments. However, there may be classes of phenomena in our sensory processes that might be studied online where the equipment and stimuli vary. Suppose the critical feature of the stimulus is an abstraction of the physical stimulus that does not vary with different displays. In that case, these features can be successfully studied online, meaning that results from online studies will match those from controlled laboratories. This study will examine the Ebbinghaus illusion to illustrate the successful use of the web for perceptual research. The implications and some discussion of types of perceptual studies conducted on the web will be discussed.
\end{abstract}

Keywords: web research, Ebbinghaus illusion, sensation and perception, technical variance, invariance

The use of the web for psychological research has become commonplace for many areas of psychology, often with success, while it is important to acknowledge the methodological issues that need to be considered (Birnbaum, 2021; Krantz \& Reips, 2017). However, the study of sensation and perception has not taken advantage of the web with the same vigor as many other fields of psychology. The inability to control stimuli online is a major drawback to studying these issues online (Birnbaum, 2021; Krantz, 2001). The inability to assure that the display and the surrounding environment remain constant is a significant drawback for these studies. Stimuli will vary in size, contrast, and wavelength composition across devices that are used by participants on the web. The viewing distance and lighting environment adds to this variation. These factors can be significant in altering the quality and even the nature of the stimulus. Most crucially, the independent variable can be altered from what was intended by the experimenter. For example, in a stimulus-dependent upon the relative light levels across the stimulus, contrast, these relative light levels can be altered significantly by environmental lighting (Krantz et al., 1992).

On the other side of the debate are arguments that the equipment variation might help such research, a position known as technical variance (Reips, 2000; Schmidt, 2007). Thus, if the study results hold over the variation of equipment, and we should include environments, though measuring these variables is hard. The results have stronger external validity, arguing that the variation of equipment and environments is expected in the world we encounter. It is probable that the phenomenon under study will determine if the range of technical variance on the web will allow for a viable study. Honing (2021) discusses how using a gaming methodology can take advantage of the technical variance in studying music perception.

It seems fitting in a journal that Ebbinghaus founded to report if the Ebbinghaus illusion (Ebbinghaus, 1902) replicates online to test if visual results can be obtained with sufficient robustness in this experimental environment. In the Ebbinghaus illusion, the participant compares two circles, shown in Figure 1, and judges the relative size of the two central circles. To give some terminology, the crucial circles are the central circles on each side forming the complex. The surrounding circles (or inducers) are manipulated to alter the perception (Figure 1). In the classic illusion, the center circle on the left looks larger than the center circle on the right, even though the two circles are objectively the same size. An online example can be found in Michael Bach's excellent collection of illusions (Bach, 2021).

Because this illusion is about the perception of size, of all the ways that the web can alter the stimuli, the differences in monitor size and image area size in the experiment will have the biggest potential impact on results, as long as contrast on the monitor reaches a basic minimum level (Krantz et al., 1992). But of interest is that the relative size of the inducers and the center circles can be generated to render 


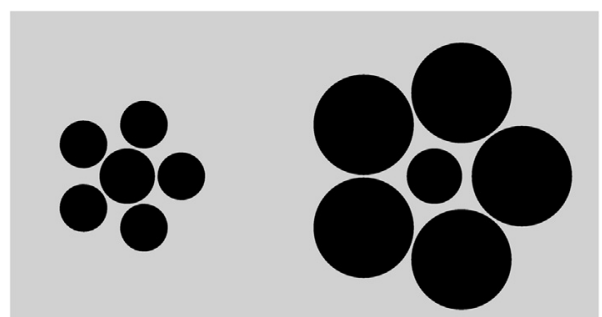

Figure 1. An example of the Ebbinghaus illusion. The center circles have the same size, but most people see the circle on the left as larger.

them constant across all these variations. One interpretation of the illusion argues that the perception of size is relative and not absolute (Massaro \& Anderson, 1971, Tozawa, 2010). Because the proportions of the stimuli can be drawn to remain the same regardless of the monitor situation, it seems probable that this illusion will work in the online environment. The inclusion of this illusion in Michael Bach's collection is suggestive though no data seems to have been collected on participants' responses to his demonstration of the illusion.

Returning to the issue of technical variance, it is possible that studying a sensory, in this case, a visual phenomenon on the web, could change our understanding of how such a phenomenon is processed. The world, and the web to a lesser extent, is a constantly changing environment (Gibson, 1986; Krantz, 2001). Our sensory systems operate in such environments quite successfully. Phenomena that are successfully studied on the web will give us hints about variables that are important to sensory processing.

\section{Method}

\section{Stimuli}

The Ebbinghaus illusion was run using the layout showing in Figure 1. The left-hand part of the stimulus setup is the standard and did not change for the participant. It was $5 \%$ of the height of the drawing canvas. The right-hand stimulus was the comparison. The diameter of the center circle on the right varied relative to the circle's diameter on the left-hand side from half to double the size in 9 logarithmically equallysize steps. This variable will be called the center ratio and was a within-subject factor used to determine the extent of the illusion.

Normally in perception studies, the designs are completely within-subject to allow cleaner interpretation of results and clearer examination of the impact of independent variables on results. However, pilot tests suggested that more participants complete the study if one of the independent variables was made a between-subject variable. Thus, the other independent variable, the relative size of the inducers or surround circles to the left-hand center circle, was a between-subject variable. This variable is called the outer ratio. There were three conditions: 0.5 , 0.75 , and 1 . The size of the left-hand outer circles where the inverse of the right-hand outer circles. Thus, in the 0.5 condition, the size of the right-hand inducers was half the size of the left-hand center circle, and the left-hand outer circles were double the size of the left-hand center circle. Figure 2 illustrates these two independent variables on one of the stimuli used in the experiment.

\section{Program}

The experiment was JavaScript/html5 using canvas for the drawing platform written by the experimenter (to access the software and data, please follow https://psychology.hanover.edu/downloads/EbbinghausSoftware.zip). The stimuli were drawn dynamically during the experiment. The size of the window in the browser was read by the program, and the canvas and stimuli were all drawn in proportion to the width of the screen. The canvas filled the entire width of the browser window, as was $92 \%$ the height of the visible browser window. The rest of the height was used for the response buttons. The study can be found at http:// psych2.hanover.edu/research/sizes/.

\section{Procedure}

Participants first read an informed consent form. Clicking to continue was expressed as giving consent. Then participants filled out a brief demographics form. Next, participants clicked a button to open a window that filled their screen with the Ebbinghaus stimuli in the middle. At the bottom of the screen, participants were asked which side had the larger center circle with a button to indicate the left or the right stimuli. Each participant was in one of the outer ratio conditions.

In this study, the method of constant stimuli was used to present the stimuli. The nine levels of center ratio were randomly presented 16 times to create 144 trials for each participant. The participant activated one of two buttons to indicate if the left or right center circle looked larger. The buttons could be clicked or touched to activate. Trials were not timed. After pressing the button, the next trial was presented. At the end of the session, participants were sent to a debriefing form where the experiment was further explained.

The participant's responses on each trial were collected, which will give a psychometric function that shows the percentage of times the participant thinks the center circle 


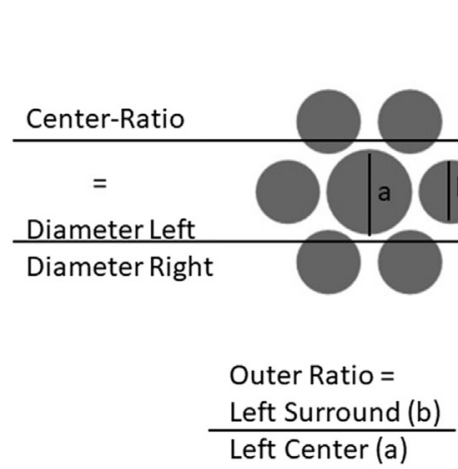

on the right is larger as a function of the center ratio. The size of the illusion is determined by the relative diameter of the right-hand center circle, where participants reported that the right-center circle was larger $50 \%$ of the time, which also means that they reported the left-hand center circle larger $50 \%$ of the time.

\section{Participants}

This study had 47 participants (self-identified as: Female 25, Male 17, Nonbinary 1, Genderqueer 1, with 3 not reporting). The mean age was 31.4 years, with a large positive skew (two did not report age). A seriousness check was given, with most participants reporting that they were serious in taking the study (Aust et al., 2013; Reips, 2000, 2009). However, no significant difference in the data was found due to the seriousness check, though the power was low, so data from all participants were used in the analyses. While five participants did not complete the entire study, they completed enough that their results were comparable and used in the final analysis. There were 15 participants in the $0.5,20$ in the 0.75 , and 12 in the 1.0 center circle ratio conditions.

\section{Results}

The percentage of time that they thought the right-hand center circle was larger was determined for each participant. By having a full psychometric function, it is possible to examine the size of the illusion and how the perception of size changes during the region of unstable perception giving us a better sense if the perception is consistent. Figure 3 plots the average percentage of presentations on which the participants said the circle on the right was larger than that on the left, with a separate curve for each condition in which the outer circles on the right were $0.5,0.75$, or 1.0 times as large as the center circle on the left. The center ratios are on the $x$-axis and the proportion of times the participants reported that the right-hand circle was larger is on
Figure 2. The two independent variables manipulated in this experiment. The within-subject variable is the relative size of the left-hand center circle to the right-hand center circle. The between-subject variable was the relative size of the surround-circles to the left-hand center circle.

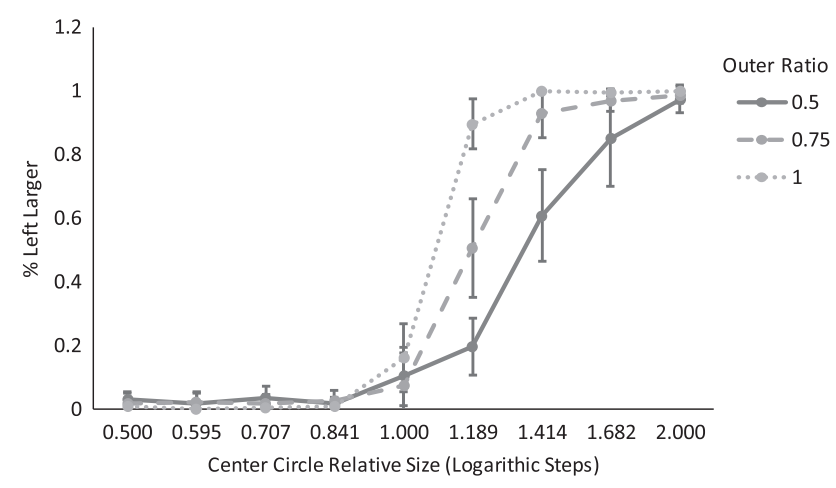

Figure 3. The results of the Ebbinghaus illusion experiment. The percentage of times a participant thought the left-hand circle was larger is plotted as a function of the relative size of the two center circles. The different lines indicate the relative sizes of the outer circles, solid for an outer ratio of 0.5 , the large dashes for an outer ratio of 0.75 , and small dashes for an outer ratio of 1 . The error bars are $95 \%$ confidence intervals.

the $y$-axis. The error bars are 95\% confidence intervals (CI). The results appear to be interpretable without the benefit of a statistical analysis given the relatively small error bars with no overlap in the region of interest in the data where participants showed uncertainty about which center circle was larger. Visually, the figure suggests that the illusion gets stronger as the outer ratio gets farther from 1 . This conclusion can be confirmed when the size of the illusion is estimated from the data. Using the non-parametric method of Żychaluk and Foster (2009), when the outer ratio is 0.5, the illusion was that the right-hand circle had to be $37 \%$ [CI: $34 \%-40 \%$ ] larger to be perceived the same size as the left-hand circle. The comparable ratio for the outer ratio of 0.75 was $19 \%$ [CI: $18 \%-20 \%$ ], and for the outer ratio of 1.0 was $8 \%$ [CI: 7\%-9\%]. From the confidence intervals, it is clear that each condition leads to a distinct size of the illusion.

The illusion described above is seen that as the outer ratio gets father from 1 , the illusion is larger. If the participants thought the two circles were the same size, this value should be nearer $50 \%$ if the center-circle ratio was 1 . As the size of the surrounding circles gets farther from 1 (same size 
as left-center circle), the size of the illusion grows, as seen by the curve rising to cross the $50 \%$ line at greater values of center circle relative size.

The relatively small error bars, see, for example, Knol and colleagues (2015), and lack of overlap of the functions in the crucial area where people are not precise about judging which center circle is larger is of note and as one of the characteristics sought in careful psychophysical results. The fact that the data is this robust under the variations of conditions is worthy of note.

\section{Discussion}

The results show a robust Ebbinghaus illusion that is sensitive to the size of the surrounding circles. Participants tended to judge that the center circle with the smaller sounds was larger than it was and the opposite if the surrounding circles were larger. The size of the illusion found here is at least as strong as seen in some other studies. Doherty and colleagues (2010) found an illusion of about $10 \%$ in adults when the surround ratio was about 0.8 . The closest condition here was a surround ratio was 0.75 , and the illusion was estimated to be $19 \%$. Massaro and Anderson (1971) found an about 6\% illusion when the surround ratio was smaller than the 0.5 value used here. This value is much smaller, but they only had surround circles on one of the center circles, and they used the method of adjustment, which might account for some of the differences.

These findings come despite several limitations imposed by the online methodology. First and foremost, the size of the stimuli varied greatly across participants. However, the relative sizes of the circles and the surrounding circles in the displays are preserved in the Web presentation, even if the screen sizes differ. It is even possible that some participants used a phone, which would render the size quite small, but tablets, desktops, and laptops were all used with varying sizes of screens, resolutions, intensities, contrasts, and many other variations. Other issues to note that could limit the power of this study are the use of inexperienced observers, a design where the main independent variable was between subjects, and the sample size. In this case, the technical variance argument holds for the interpretation of the results and that the relative size of the stimuli is the overall importance to the interpretation of the results.

The results can be examined with two different theoretical ideas. First, in J. J. Gibson's (1986) Ecological Perception Theory, perception is driven by the extraction of invariants from the world. Gibson was not very impressed with the great variation in the retinal image. This variation is endemic to the experience of the world and not instructive to how perception works. According to this the- ory, the visual system's job is to scan the retinal image for those features that do not change as the retinal image differs. These are the invariants. Many studies of the Ebbinghaus illusion examine the absolute size of the different circles in examining the size of the illusion (e.g., Massaro \& Anderson, 1971). Even if the physical or perceived size is not considered the most important feature, retinal image size is considered the most important factor in the illusion (Takao et al., 2019). In the current study, the absolute sizes of the circles vary for each participant, so if the absolute size is most important, the results should show such a large variation that the results would be uninterpretable. The current results suggest that the invariance of the relative size of the outer circles to the inner circles is crucial to the perception of the Ebbinghaus illusion and, thus, allows for the robust findings reported here. This study did not examine the actual size of circles, so the actual or even the retinal image size might still be important factors, but given that the results are at least as strong as seen in some published studies, it raises the possibility that relative size plays the role of an invariant here (Doherty et al., 2010; Massaro \& Anderson, 1971).

The other theoretical idea is that of relational theory (Tozawa, 2010). The relational theory specifies that the perceived size of objects is determined by how much of a texture is covered by an object. In this illusion, there is no texture, but if the theory can be expanded to consider the surrounding objects as a texture instead of covering texture. Applying this theory to the Ebbinghaus illusion would be due to the relative size of the surround to the center circles. This theory also applies to suggest that the illusion should work consistently across varied screens with similar strength to a carefully controlled experimental situation. It is unnecessary to see these as competing ideas, but the role of the texture and relative size could be the invariants extracted in this stimulus configuration.

When the idea of technical variance (Reips, 2000; Schmidt, 2007) is usually discussed, it suggests that the variations of the web lead to greater ecological validity. While that is true in the present experiment, technical variance also suggests a potential for modification of the theoretical understanding of the phenomenon. By allowing the variation of the equipment, variables that survive the technical variance are those that can be studied, e.g., relative size of the surround circles, the inducers, to the center circles. If such findings can be replicated and extended, it could shift the theories developed for some sensory phenomena.

From this discussion, it is necessary to consider which stimuli survive the technical variance of online research and which do not. Clearly, from the example of the Ebbinghaus illusion, it would seem that stimuli and visual phenomena based on high contrast images where the effect 
is dependent upon relationships within the stimuli ought to be successfully studied online. Thus, those phenomena that use relational judgments where the participant needs to judge among two or more stimuli and the relationship is not altered by size or resolution of the monitor, then the phenomenon should be studied online. If we use the standard separation of sensation and perception, there might be another way to make predictions. The sensation is traditionally thought to study the basic reception of sensory information, brightness, color, and so forth. Perception is then thought to process the complete stimulus and provide identification and even meaning. Given the greater richness of stimuli used in perception research, it could be argued that perception is possible to study on the web, but the sensation is not with its simpler stimuli where the values change across equipment. The one caveat is that the critical feature of the stimuli to be perceived should not be near any contrast thresholds as this could call important features above and below contrast on different equipment (Krantz, 2001). Such problems have been found in clinical situations where subtle differences in shading can hide clinically relevant features of the image (Krantz, 2008).

The role of consistent relationships within the stimuli is perhaps part of the way the visual system operates. Zeki (1993) discusses the role of more advanced processing of the visual cortex where the physical values are no longer relevant, but the relationship within the stimulus is relevant. Zeki was discussing color constancy, or how we see different objects as the same color despite great changes in the illumination, which changes the wavelengths of the light entering the eye. Following this principle shows that the need for fixed relationships is important not just in web research but also in vision in general.

\section{Conclusion}

In summary, perception research is possible online (see Honing, 2021). Robust results can be obtained if the feature being studied does not depend upon the particular device screen and all its associated characteristics. Many studies that fall in the domain of perception probably can be attempted. The subtler the display characteristics are or, the more sensitive to environmental and display variations, the less likely the study will be successful. In most of these cases, have a laboratory study as a comparison is advisable until the perceptual characteristics that survive the technical variance of the web are better known.

\section{References}

Aust, F., Diedenhofen, B., Ullrich, S., \& Musch, J. (2013). Seriousness checks are useful to improve data validity in online research. Behavior Research Methods, 45(2), 527-535. https:// doi.org/10.3758/s13428-012-0265-2
Bach, M. (2021, May 12). 143 Optical Illusions \& Visual Phenomena. https://michaelbach.de/ot/index.html

Birnbaum, M. H. (2021). Advanced training in web-based psychology research: Trends and future directions. Zeitschrift für Psychologie, 229(4), 260-265. https://doi.org/10.1027/21512604/a000473

Ebbinghaus, H. (1902). The principles of psychology. Veit.

Doherty, M. J., Campbell, N. M., Tsuji, H., \& Phillips, W. A. (2010) The Ebbinghaus illusion deceives adults but not young children. Developmental Science, 13(5), 714-721. https://doi.org/ 10.1111/j.1467-7687.2009.00931.x

Gibson, J. J. (1986). The ecological approach to visual perception. Erlbaum.

Honing, H. (2021). Lured into listening: Engaging games as an alternative to reward-based crowdsourcing in music research. Zeitschrift für Psychologie, 229(4), 266-268. https://doi.org/ 10.1027/2151-2604/a000474

Knol, H., Huys, R., Sarrazin, J. C., \& Jirsa, V. K. (2015). Quantifying the Ebbinghaus figure effect: Target size, context size, and target-context distance determine the presence and direction of the illusion. Frontiers in Psychology, 6, Article 1679. https:// doi.org/10.3389/fpsyg.2015.01679

Krantz, J. H. (2001). Stimulus delivery on the web: What can be presented when calibration isn't possible. In U.-D. Reips \& M. Bosnjak (Eds.), Dimensions of Internet Science (pp. 113-130). Pabst Science.

Krantz, J. H. (2008). Did I really see that? The complex relationship between the visual stimulus and visual perception. Journal of Voice, 22(5), 520-532. https://doi.org/10.1016/j.jvoice.2007.02.003

Krantz, J. H., Ballard, J., \& Scher, J. (1997). Comparing the results of laboratory and World-Wide Web Samples on the determinants of female attractiveness. Behavior Research Methods, Instruments, \& Computers, 29, 264-269. https://doi.org/ 10.3758/BF03204824

Krantz, J. H., \& Reips, U.-D. (2017). The state of web-based research: A survey and call for inclusion in curricula. Behavior Research Methods, 49(5), 1621-1629. https://doi.org/10.3758/ s13428-017-0882-x

Krantz, J. H., Silverstein, L. D., \& Yeh, Y. (1992). Visibility of transmissive liquid crystal displays under dynamic lighting conditions. Human Factors, 34, 615-632. https://doi.org/ $10.1177 / 001872089203400508$

Massaro, D. W., \& Anderson, N. H. (1971). Judgmental model of the Ebbinghaus illusion. Journal of Experimental Psychology, 89(1), 147-151. https://doi.org/10.1037/h0031158

Reips, U.-D. (1997). Das psychologische Experimentieren im Internet [Psychological experimenting on the Internet]. In B. Batinic (Ed.), Internet für Psychologen (pp. 245-265). Hogrefe.

Reips, U.-D. (2000). The Web experiment method: Advantages, disadvantages, and solutions. In M. H. Birnbaum (Ed.), Psychological experiments on the Internet (pp. 89-117). Academic Press.

Reips, U.-D. (2009). Internet experiments: Methods, guidelines, metadata. In B. E. Rogowitz \& T. N. Pappas (Eds.), Human vision and electronic imaging XIV (Vol. 7240, p. 724008). International Society for Optics and Photonics.

Schmidt, W. C. (2007). Technical considerations when implementing online research. In K. McKenna, A. N. Joinson, U.-D. Reips, \& T. Postmes (Eds.), The Oxford handbook of Internet psychology (pp. 461-472). . Oxford University Press.

Takao, S., Clifford, C. W., \& Watanabe, K. (2019). Ebbinghaus illusion depends more on the retinal than perceived size of surrounding stimuli. Vision Research, 154, 80-84. https://doi. org/10.1016/j.visres.2018.10.010

Tozawa, J. (2010). Role of a texture gradient in the perception of relative size. Perception, 39(5), 641-660. https://doi.org/ $10.1068 /$ p6440 
Zeki, S. (1993). A vision of the brain. Blackwell Scientific Publications.

Żychaluk, K., \& Foster, D. H. (2009). Model-free estimation of the psychometric function, Attention, Perception. \& Psychophysics, 71(6), 1414-1425. https://doi.org/10.3758/APP.71.6.1414

\section{History}

Received February 9, 2021

Revision received June 28, 2021

Accepted June 28, 2021

Published online December 17, 2021

\section{Acknowledgments}

The author would like to thank Chris R. Wolfe for his support, guidance, and advice on this project. Without his help, this project would not be. The author would also like to thank the reviewers and editors for their comments that have greatly improved this paper.

\section{Open Data}

The online material to the study is publicly available at http:// psych2.hanover.edu/research/sizes/. To access the software and data to the experiment, please follow https://psychology.hanover. edu/downloads/EbbinghausSoftware.zip

\section{Funding}

Open access publication enabled by Hogrefe Publishing.

\section{John H. Krantz}

Department of Psychology

Hanover College

517 Ball Drive

Hanover, IN 47243

USA

krantzj@hanover.edu 